\title{
PATH-CONNECTED CLOSURE OF UNITARY ORBITS
}

\author{
DON HADWIN AND WENJING LIU
}

\begin{abstract}
If $\mathscr{A}$ and $\mathscr{B}$ are unital $\mathrm{C}^{*}$-algebras and $\pi: \mathscr{A} \rightarrow \mathscr{B}$ is a unital $*$-homomorphism, then $\mathscr{U}_{\mathscr{B}}(\pi)^{-}$is the set of all $*$-homomorphisms from $\mathscr{A}$ to $\mathscr{B}$ that are approximately (unitarily) equivalent to $\pi$. We address the question of when $\mathscr{U}_{\mathscr{B}}(\pi)^{-}$is path-connected with respect to the topology of pointwise norm convergence. When $\mathscr{A}$ is singly generated and $\mathscr{B}=B\left(\ell^{2}\right)$, an affirmative answer was given in [4]; we extend this to the case when $\mathscr{A}$ is separable. We also give an affirmative answer when $\mathscr{B}$ is a von Neumann algebra and $\mathscr{A}$ is $\mathrm{AF}$ or homogeneous; if $\mathscr{B}$ is finite $\mathscr{A}$ need only be ASH.
\end{abstract}

Mathematics subject classification (2020): Primary 46L05; Secondary 47C15.

Keywords and phrases: $\mathrm{C}^{*}$-algebra, representation, unitary orbit.

\section{REFERENCES}

[1] B. BlackADAR, K-theory for operator algebras, second edition, Mathematical Sciences Research Institute Publications, 5. Cambridge University Press, Cambridge, 1998.

[2] K. R. DAvidson, $C^{*}$-algebras by example, Fields Institute Monographs, 6. American Mathematical Society, Providence, RI, 1996.

[3] H. Ding AND D. Hadwin, Approximate equivalence in von Neumann algebras, Sci. China Ser. A 48 (2005), no. 2, 239-247.

[4] D. HADwIN, An operator-valued spectrum, Indiana Univ. Math. J. 26 (1977), no. 2, 329-340.

[5] D. HADWIN AND W. LiU, Approximate equivalence in von Neumann algebras, preprint, 2020.

[6] R. V. KADISON AND J. R. Ringrose, Fundamentals of the theory of operator algebras, Vol. II, Advanced theory, Corrected reprint of the 1986 original. Graduate Studies in Mathematics, 16. American Mathematical Society, Providence, RI, 1997.

[7] C. Olsen And W. Zame, Some $C^{*}$-algebras with a single generator, Trans. Amer. Math. Soc. 215 (1976), 205-217.

[8] M. RøRdam, F. LaRsen AND N. LaUstSen, An introduction to $K$-theory for $C^{*}$-algebras, London Mathematical Society Student Texts, 49, Cambridge University Press, Cambridge, 2000.

[9] D. SHERMAN, Unitary orbits of normal operators in von Neumann algebras, J. reine angew. Math. 605 (2007) 95-132.

[10] D. Sherman, On Cardinal invariants and generators for von Neumann algebras, Canad. J. Math. 64 (2012) 455-50. 